\title{
Beispiele von Einsätzen der DEZA im Dezentralisierungsprozess
}

\section{Pascal Raess}

\section{(2) OpenEdition}

\section{Journals}

Electronic version

URL: http://journals.openedition.org/sjep/416

DOI: $10.4000 /$ sjep.416

ISSN: 1663-9677

\section{Publisher}

Institut de hautes études internationales et du développement

\section{Printed version}

Date of publication: 1 novembre 2007

Number of pages: $257-258$

ISBN: 978-2-88247-069-0

ISSN: 1660-5926

Electronic reference

Pascal Raess, " Beispiele von Einsätzen der DEZA im Dezentralisierungsprozess », Schweizerisches Jahrbuch für Entwicklungspolitik [Online], 26-2 | 2007, Online erschienen am: 03 Juni 2010, abgerufen am 08 September 2020. URL : http://journals.openedition.org/sjep/416 ; DOI : https://doi.org/ $10.4000 /$ sjep.416 


\title{
Beispiele von Einsätzen der DEZA im Dezentralisierungsprozess
}

\author{
Pascal Raess
}

\section{Benin: Hilfe für die Festigung des Lokalstaates im Dezentralisierungsprozess ${ }^{1}$}

Die Dezentralisierungspolitik wurde in Benin in der Verfassung von 1990 verankert und wird seit den Kommunalwahlen von Dezember 2002 und Januar 2003 über einen Gesetzesrahmen umgesetzt, welcher im Wesentlichen staatliche Dienstleistungen auf die Departement- und Gemeindeebene verlagern, Kompetenzen und Ressourcen an die kommunalen und darunter liegenden Organe übertragen und das öffentliche Dienstleistungsangebot verbessern soll, indem die Bedürfnisse der Bevölkerung tatsächlich berücksichtigt werden. Zu den Hauptproblemen gehörte, dass die Abgeordneten ihre Rolle meistern konnten und die vorhandenen lokalen Kräfte koordinierten, um konkrete Resultate zu erzielen, und dass menschliche und finanzielle Ressourcen fehlten. Obwohl die Gemeinden die Eigentümer der meisten öffentlichen Bauten sind, beteiligen sie sich kaum an deren Management (Wartung, Instandhaltung). Das Konzept service public ist noch wenig bekannt.

Die DEZA engagiert sich seit 1997 mit verschiedenen Unterstützungsleistungen in der Vorbereitung der Dezentralisierung und im Aufbau der lokalen Entwicklung. Das Programm „Appui au renforcement de l'Etat local" (Unterstützung zur Stärkung des Lokalstaates) verfolgt als Ansatz die Entwicklung der Gebiete, die Förderung der Basisdemokratie und die Unterstützung der infrakommunalen Ebene. Ziel ist es, die Lebensbedingungen der Frauen, Männer und Kinder zu verbessern, Mechanismen zur besseren Beteiligung der Akteure der Zivilgesellschaft bei der Definition der lokalen öffentlichen Politik zu fördern und die Rolle der Gemeinde als Motor für die lokale Entwicklung zu konsolidieren. Das Programm erschliesst Synergien mit andern Programmen der DEZA (z.B. Ausbildung oder lokale Wirtschaft). Das Hauptziel lässt sich wie folgt zusammenfassen: Die Zivilgesellschaft, die Wirtschaftsbeteiligten und die Gemeinden einigen sich in den „Entwicklungsterritorien" auf qualitativ hochstehende Dienstleistungen in ausreichender Menge, welche den Bedürfnissen der Bevölkerung entsprechen. Dieses ehrgeizige Programm soll über die staatlichen Mechanismen umgesetzt und nach den Mechanismen des öffentlichen Finanzmanagement finanziert werden. Die Frage der Finanzierung dieser Dienstleistungen von angemessener Quantität und Qualität steht demnach im Mittelpunkt des Programms.

\section{Mosambik: Programm zur Förderung der Dezentralisierung und der Gemeindeautonomie}

Dezentralisierung, Partizipation und eine effiziente Lokalregierung bilden die Hauptanliegen in den Anstrengungen Mosambiks, die Armut substanziell zu verringern und zur Verwirklichung der MDG beizutragen. Der langfristige Aktionsplan für eine beschleunigte Armutsverringerung (PARPA) der Regierung Mosambiks sowie der ergänzende Plan für die Reform des öffentlichen Sektors lassen klar ersehen, dass die Dezentralisierung und die Dekonzentration, aus der effizientere und transparentere Lokalregierungen hervorgehen, eine notwendige Voraussetzung für basisnahe Dienstleistungen an die armen Bevölkerungsschichten und für Arbeitsplätze und Einkommen auf lokaler Ebene bilden. Diese Herausforderung bleibt angesichts der Geschichte Mosambiks Kolonialzeit, Nachkolonialzeit, Nachkonfliktzeit enorm; das Land ist geprägt durch eine stark

1 Die Informationen über Benin, Mosambik und Pakistan stammen aus internen Unterlagen der DEZA, diejenigen über Bolivien gehen auf die folgende Veröffentlichung zurück: L. Thévoz, „La décentralisation dans les Andes ou l'art d'accompagner un processus", Politorbis. Revue de politique étrangère, Nr. 41, 2/2006, Bern, EDA, 2006, S. 43-50. 
zentralisierte öffentliche Verwaltung und eine mangelhafte Ausstattung der lokalen Ebene mit finanziellen und menschlichen Ressourcen. Die Regierung und das Parlament haben jedoch in den frühen 90er-Jahren wichtige Weichenstellungen mit Blick auf die Dezentralisierung getroffen.

Die DEZA hat die wichtige Rolle der Dezentralisierungsvorhaben anerkannt, als sie im Jahr 2002 das innovative Programm für lokale Governance namens "Programme de soutien à la décentralisation et la municipalisation (Programm zur Unterstützung der Dezentralisierung und Kommunalisierung) startete. Dieses Programm konzentriert sich namentlich auf die folgenden Aspekte: Verbesserung der Planungsverfahren, besonders im Budgetbereich, durch grössere Partizipation; Kapazitätsaufbau zwecks grösserer Transparenz und Rechenschaftspflicht der lokalen Steuerverwaltung; Verbesserung des Angebots und des Zugangs zu öffentlichen Dienstleistungen, vor allem für die marginalisierten Bevölkerungen; Beitrag an den nationalen politischen Dialog über die Politik und die Umsetzung der Dezentralisierung im Steuersektor.

\section{Bolivien: Unterstützung des \\ Dezentralisierungsprozesses}

Der Dezentralisierungsprozess in Bolivien weist eine Reihe grösserer Innovationen auf, die in diesem Bereich Meilensteine setzten, darunter insbesondere die "Schockmethode" (in Bolivien wurde am 1. Juli 1994 ohne jegliche Pilotprojekte praktisch über Nacht die Gemeindeautonomie eingeführt), die Institutionalisierung der Kontrolle des Volkes über das lokale Management (die traditionellen gesellschaftlichen Organisationen wurden anerkannt und erhielten ein echtes Einsichtsrecht, sodass sie sich zu unverzichtbaren Partnern der gewählten Kommunalbehörden entwickelten), der direkte Transfer von nationalen Finanzressourcen an die Kommunen sowie die allgemeine Einführung eines partizipativen Haushalts.

Die DEZA unterstützt generell Reformen des Staates, die einen deutlich spürbaren Beitrag an die Armutsverringerung leisten können. Die Dezentralisierung gehört dazu, weil sie direkt das Empowerment der lokalen Akteure und nach
Möglichkeit der ärmsten unter ihnen fördert, die Partizipation der lokalen Akteure im Entscheidungsprozess vorantreibt und dazu führt, dass die Begünstigten die Reformen selbst in die Hand nehmen. Zusammen genommen verbessern die Reformen den Zugang der ärmsten Bevölkerungsschichten zu öffentlichen Dienstleistungen, die ihren Bedürfnissen und ihren Mitteln entsprechen.

\section{Pakistan: innovatives Programm ETR}

Dieses Projekt der DEZA soll in Pakistan im Kontext der Dezentralisierung einen Prüfstein für den Einsatz von fiskalischen Instrumenten zur Bekämpfung von Armut und Umweltzerstörung bilden. Da das Instrument neu ist, muss die Bevölkerung sensibilisiert und für zwei Grundsätze - das Verursacherprinzip und das Gerechtigkeitsprinzip - gewonnen werden. Die ärmsten Bevölkerungen leiden in der Tat am meisten unter Umweltkatastrophen und unter der Zerstörung der Ökosysteme. Diese gehen auf Defizite in der Wirtschafts- und Sozialpolitik zurück, die sich korrigieren lassen.

Es wurde beschlossen, dieses Instrument im Prozess der dezentralisierten Governance einzusetzen. Ziel ist es, den Dezentralisierungsprozess durch den Einsatz von fiskalischen Instrumenten zu festigen, die der Umwelt nützen (Umstellung von Verhaltensweisen) und die Armut verringern. Dies setzt nicht in erster Linie neue Steuern oder Abgaben voraus, sondern Recherchen zur Machbarkeit der verschiedenen ETR-Instrumente in Pakistan und zu deren Umsetzung in Pilotprojekten. Spezifischer gesehen verfolgt das innovative Projekt die folgenden Ziele: Akzeptanz für das „Verursacherprinzip" auf Bezirksebene schaffen, Machbarkeit der Umsetzung verschiedener Optionen in Bereichen wie Kosten von Gesundheit, Ausbildung, Wohnungen, Wasser prüfen, die Kapazitäten für die Verwendung von fiskalischen Instrumenten und für die Bildung neuer Koalitionen mit der Zivilgesellschaft zur Thematik verbessern. Die Resultate bestehen demnach nicht in erster Linie in der Zunahme der Steuereinnahmen, sondern in der Schaffung eines förderlichen Rahmens, damit fiskalische Instrumente im Interesse der Umwelt und der Armutsverringerung eingeführt und verwendet werden können. 